\title{
Correction to "Winter interactions between weather regimes and marine surface in the North Atlantic European region"
}

\author{
Virginie Guemas, David Salas-Mélia, Masa Kageyama, Hervé Giordani, Aurore Voldoire, \\ and Emilia Sanchez-Gomez \\ Received 12 January 2010; published 3 April 2010.
}

Citation: Guemas, V., D. Salas-Mélia, M. Kageyama, H. Giordani, A. Voldoire, and E. Sanchez-Gomez (2010), Correction to "Winter interactions between weather regimes and marine surface in the North Atlantic European region," Geophys. Res. Lett., 37, L07806, doi:10.1029/2010GL042485.

[1] In the paper "Winter interactions between weather regimes and marine surface in the North Atlantic European region" by Virginie Guemas et al. (Geophysical Research Letters, 36, L09816, doi:10.1029/2009GL037551), the statistical test chosen to estimate the significance level of the difference between the persistence of a regime in a sensitivity experiment and in the control experiment underestimated the uncertainty on this difference. The resulting decision making procedure rejects too frequently the null hypothesis $\mathrm{H}_{\mathrm{o}}$ that this difference equals 0 . A new bootstrap test has therefore been developed.

[2] The mean persistence of a weather regime in an experiment is computed 1000 times by drawing randomly and with replacement the same number of values of persistence than the sample size, where the sample size is the number of weather regime episodes. Then, 1000 differences are computed between the 1000 persistence values in the sensitivity experiment and the 1000 ones in the control experiment. These 1000 differences allow to build a histogram and thus to estimate the distribution of the difference between the persistence of the regime in the sensitivity experiment and its persistence in the control. From this distribution, we can perform a two-sided test of hypothesis to assess if this difference is equal to zero or not.

[3] To state whether the changes in mean persistence induced by sea surface temperature anomalies and sea ice concentration anomalies are statistically relevant or not, the set of 5 experiments consisting of an ensemble of 50 simulations of the winter (December-February) season was complemented by another set of ensembles of 50 simulations. From the 5 experiments consisting each of 100 members, the significance level of the difference between the persistence of a regime in a sensitivity experiment and its persistence in the control can be computed according to the test described above.

[4] The only change in mean persistence which reaches the $90 \%$ significance level is the decrease in NAO- persistence by 0.61 days when the atmosphere is forced with the pattern of SST and sea ice concentration induced by the NAO- regime instead of the climatology (CTL). The $\mathrm{p}$ _value is 0.08 . We conclude that the interaction with the marine surface could induce a negative feedback onto the NAOregime persistence. However, the results about the role of the interaction with the marine surface on transitions from the Zonal toward the Atlantic Ridge regime and from the Blocking toward the Atlantic Ridge regime do not seem to be robust. 